\title{
Nonalcoholic Steatohepatitis: A Proposal for Grading and Staging the Histological Lesions
}

\author{
Elizabeth M. Brunt, M.D., Christine G. Janney, M.D., Adrian M. Di Bisceglie, M.D., \\ Brent A. Neuschwander-Tetri, M.D., and Bruce R. Bacon, M.D. \\ Department of Pathology and Division of Gastroenterology and Hepatology, Department of Internal \\ Medicine, Saint Louis University School of Medicine, St. Louis, Missouri
}

OBJECTIVE: Steatohepatitis is a morphological pattern of liver injury that may be seen in alcoholic or nonalcoholic liver disease. This pattern may occur with obesity, diabetes, the use of certain drugs, or the cause may be idiopathic. The well-recognized histopathological features of nonalcoholic steatohepatitis (NASH) include hepatocellular steatosis and ballooning, mixed acute and chronic lobular inflammation, and zone 3 perisinusoidal fibrosis. Currently, there are no systems for grading necroinflammatory activity or for staging fibrosis as exist for various other forms of chronic liver disease. The purpose of this study was to develop such a grading and staging system and was based on review of liver biopsies from 51 patients with nonalcoholic steatohepatitis from Saint Louis University Health Sciences Center.

METHODS: For determination of grade, 10 histological variables of activity were initially analyzed; an overall impression of mild, moderate, and severe was made and the variables considered to be most significant were used to develop the necroinflammatory grade.

RESULTS: The histological lesions considered to be significant were: steatosis, ballooning, and intra-acinar and portal inflammation. A staging score was developed to reflect both location and extent of fibrosis. The fibrosis score was derived from the extent of zone 3 perisinusoidal fibrosis with possible additional portal/periportal fibrosis and architectural remodeling. Fibrosis stages are as follows: Stage 1, zone 3 perisinusoidal fibrosis; Stage 2, as above with portal fibrosis; Stage 3, as above with bridging fibrosis; and Stage 4, cirrhosis.

CONCLUSION: We propose a grading and staging system that reflects the unique histological features of nonalcoholic steatohepatitis. (Am J Gastroenterol 1999;94:2467-2474. (C) 1999 by Am. Coll. of Gastroenterology)

\section{INTRODUCTION}

Steatohepatitis is a morphological pattern of liver injury, which in nonalcoholic patients, may represent a form of chronic liver disease currently known as nonalcoholic steatohepatitis (NASH). NASH was first described as a clinical entity by Ludwig et al. (1). It is accepted that this pattern may occur in a variety of clinical settings including but not limited to diabetes and obesity, but in many cases the etiology is unknown (1-6). The distinctive morphological features of steatohepatitis, regardless of the clinical background, include some "alcoholic hepatitis-like" findings: steatosis, lobular inflammation, which includes polymorphonuclear leukocytes, and perisinusoidal fibrosis in zone 3 of the acinus. Other common features are hepatocellular ballooning, poorly formed Mallory's hyaline, and glycogenated nuclei (1, 2, 7-9). The clinical course is indolent in most patients, but cirrhosis is a known complication that has been reported in $7 \%(4), 15 \%(1,3)$ and $16 \%$ (8) of patients with NASH. NASH may be a cause for some cases of cryptogenic cirrhosis (5). To date, there are no clinical or histopathological "markers," which predict patients at risk for progression to cirrhosis.

The histological features of NASH have been well described and several of the individual features analyzed semiquantitatively $(2,3,7-9)$. However, there is no system for reporting of global assessment of necroinflammatory activity (grade) or extent of fibrosis with or without architectural remodeling (stage). Such systems currently are used for other forms of chronic liver disease such as chronic viral or autoimmune hepatitis (10-15), primary biliary cirrhosis (PBC) $(16,17)$, primary sclerosing cholangitis (PSC) (18), and methotrexate-induced liver injury (19). The pathological lesions of NASH are sufficiently different from these other forms of chronic liver diseases to preclude the use of any of the current systems for grading and staging. Therefore, we reviewed biopsy material from patients with NASH to develop a system for grading and staging the morphological changes for the purposes of communicating a more standard description of the lesions present and the degree of liver injury associated with them.

\section{MATERIALS AND METHODS}

A computer search of the Surgical Pathology files at Saint Louis University Hospital for in-house biopsies from one clinician (B.R.B.) with the diagnosis of "steatohepatitis" from January, 1992, to July, 1997 yielded 70 biopsies from 69 patients. Biopsies had been obtained after thorough clin- 
ical evaluation and signed informed consent by each patient. The protocol used in this study conformed to the guidelines of the Institutional Review Board of Saint Louis University School of Medicine. Inclusion criteria for the study were that the initial assessment of the biopsy material by the pathologist and the clinical data by the clinician concurred with the diagnosis of NASH. Specifically, exclusions were made for patients with known alcohol use in excess of $20 \mathrm{~g}$ per day, confirmed by the referring physician and a family member. In addition, biopsies from patients with other known causes of liver disease, hepatitis C seropositivity, granulomatous liver disease, hereditary hemochromatosis, alpha-1-antitrypsin deficiency, and known use of methotrexate, tamoxifen, and corticosteroids were excluded from this analysis. With the exception of alpha-1-antitrypsin deficiency, the excluded entities may, by themselves, result in steatosis, inflammation, and fibrosis. Patients with diabetes $(\mathrm{n}=3)$ and serological ANA $<1: 160(\mathrm{n}=3)$ but no evidence of autoimmune liver disease were included. The remaining 44 patients had no other known clinical conditions that would result in steatohepatitis. A total of 51 biopsies from 50 patients ( 28 women, 22 men, ages $18-72$ yr) were included in the review.

Each biopsy was analyzed by a single pathologist (E.M.B.) with three hematoxylin and eosin sections and the periodic-acid Schiff with diastase stain for the necroinflammatory grading. The Masson's trichrome and Sweet's reticulin stains were reviewed for fibrosis and architectural changes. The Perls' Prussian blue stain was used to grade iron storage.

Ten histological variables commonly described in NASH were analyzed for purposes of determining which of them were significant for necroinflammatory activity as follows:

1. Macrovesicular steatosis was graded $0-3$ based on percent of hepatocytes in the biopsy involved ( 0 is none; 1 is up to $33 \%$; 2 is $33-66 \%$; 3 is $>66 \%$ ); zonal distribution of steatosis and the presence of microvesicular steatosis were noted.

2. Hepatocellular ballooning and disarray were evaluated for zonal location, and an estimate of severity (mild, marked) was made based on numbers of hepatocytes showing this change.

3. Intra-acinar (lobular) inflammation was graded 0-3 based on inflammatory foci per $20 \times$ with a $20 \times$ ocular ( 0 ; 1 is 1 to $2 / 20 \times ; 2$ is up to $4 / 20 \times ; 3$ is $>4 / 20 \times$ ); the cellular components of inflammation (polymorphonuclear leukocytes, lymphocytes and other mononuclear cells, eosinophils and microgranulomas) and location (sinusoidal, surrounding Mallory's hyaline or hepatocellular necrosis) were noted.

4. Portal tract inflammation was graded as none, mild, moderate, and severe (0-3); types of inflammatory cells were noted (lymphocytes, plasma cells, eosinophils).

5. Mallory's hyaline was graded as $0-2$ ( 0 is absent, 1 is occasional, 2 is several); poorly formed or well-formed hyaline as well as zonal location and presence or absence of satellitosis (polymorphonuclear leukocytes surrounding liver cells with Mallory's hyaline) were noted.

6. Acidophil bodies were counted per $20 \mathrm{x}$ and graded $0-3$, as in intra-acinar inflammation.

7. PAS-D Kupffer cells were counted in a similar fashion and graded $0-3$.

8. Glycogenated nuclei were graded $0-3$ ( 0 is none, 1 is rare, 2 is several, 3 is numerous); zonal location was noted.

9. Lipogranulomas: intra-acinar lipogranulomas were noted as none, rare, occasional, or several. The cellular components of the lipogranulomas (lymphocytes, mononuclear cells, occasional single eosinophils) were noted. Larger, portal tract lipogranulomas were noted.

10. Hepatocellular iron was graded $0-4+$ as per Searle (20).

Upon completion of the separate evaluation of all of these variables, an impression-based grade of mild, moderate, or severe was assigned. Then, a numerical grade was listed based on the values recorded for the variables of steatosis + intra-acinar inflammation + portal inflammation (e.g., $2+$ $1+1)$. A working formula was derived from analyzing the impression-based grades and numerical grades, and is reported below.

Fibrosis was evaluated so as to take into account the unique zone 3 perisinusoidal fibrosis that is characteristically seen in steatohepatitis. Three categories were initially separately evaluated:

1. Perisinusoidal fibrosis was scored $0-3$ based on percent of zone 3 foci involved ( 0 is none, 1 is up to $33 \% ; 2$ is $33-66 \%$; 3 is $>66 \%$ ).

2. Portal fibrosis was scored as for staging in chronic hepatitis $0-4$ ( 0 is none; 1 is expanded portal tracts; 2 is periportal fibrosis; 3 is bridging fibrosis with nodular architecture; 4 is cirrhosis) (11-15).

3. Any form of bridging fibrosis was scored $0-4$ ( 0 is none; 1 is 1 focus; 2 is $>1$ focus with no nodularity; 3 is bridging fibrosis with nodular remodeling; 4 is cirrhosis).

These determinations were then reviewed, and a working formula was derived that reflected these forms of fibrosis to indicate the unique pattern of fibrosis in steatohepatitis. The fibrosis staging system is described below.

After completion of the biopsy analysis, the alanine aminotransferase (ALT) and aspartate aminotransferase (AST) values were recorded for the 47 patients for whom the archived clinic charts were available. The mean values were compared by the two-tailed Student's $t$ test and recorded as statistically significant at $p<0.05$. 
Table 1. Necroinflammatory Grading System for Steatohepatitis

\begin{tabular}{|c|c|}
\hline Mild, grade 1 & $\begin{array}{l}\text { Steatosis (predominantly macrovesicular) } \\
\text { involving up to } 66 \% \text { of biopsy; may see } \\
\text { occasional ballooned zone } 3 \text { hepatocytes; } \\
\text { scattered rate intra-acinar pmn's } \pm \text { intra- } \\
\text { acinar lymphocytes; no or mild portal } \\
\text { chronic inflammation. }\end{array}$ \\
\hline Moderate, grade 2 & $\begin{array}{l}\text { Steatosis of any degree; ballooning of } \\
\text { hepatocytes (predominantly zone 3) } \\
\text { obvious; intra-acinar pmn's noted, may } \\
\text { be associated with zone } 3 \text { pericellular } \\
\text { fibrosis; portal and intra-acinar chronic } \\
\text { inflammation noted, mild to moderate. }\end{array}$ \\
\hline Severe, grade 3 & $\begin{array}{l}\text { Panacinar steatosis; ballooning and } \\
\text { disarray obvious, predominantly in zone } \\
3 \text {; intra-acinar inflammation noted as } \\
\text { scattered pmn's, pms's associated with } \\
\text { ballooned hepatocytes } \pm \text { mild chronic } \\
\text { inflammation; portal chronic } \\
\text { inflammation mild or moderate, not } \\
\text { marked. }\end{array}$ \\
\hline
\end{tabular}

\section{RESULTS}

\section{Necroinflammatory Grade}

The following proposal for assigning activity grade for steatohepatitis is based on the fact that no one single feature can be used to determine activity, but rather it is the combination of features of hepatocellular steatosis, ballooning and disarray, and inflammation (acinar and portal) that determine the grade. The proposal is shown schematically in Table 1.

GRADE 1: MILD. These biopsies meet the minimum criteria for the diagnosis of steatohepatitis with some degree of hepatocellular steatosis, and characteristic lobular mixed inflammation. Intra-acinar inflammation is scattered and mild and consists of small collections of polymorphonuclear leukocytes with or without lymphocytes. One may see occasional microgranulomas and occasional lipogranulomas. Although the amount of steatosis is usually $<33 \%$ of the biopsied material, steatosis up to $66 \%$ but with only mild inflammation may be seen. The steatotic hepatocytes may be scattered throughout the lobules or concentrated in zone 3. In cases of the latter, the inflammatory component is very mild. Ballooning and disarray are minimally present, if at all. There is no or only mild portal chronic inflammation.
Portal inflammation is not seen in the absence of lobular inflammation.

GRADE 2: MODERATE. These biopsies show the combination of morphological changes described that range between those seen in mild (grade 1) and severe (grade 3). The steatosis is more predictably noted and may be seen in up to or greater than $66 \%$ of the hepatocytes. Ballooning and disarray are always present; both of these changes are predominantly seen in zone 3 hepatocytes. The ballooning is most commonly present in hepatocytes adjacent to those most distended by the steatosis. Intra-acinar inflammation, as described above, is more noticeable and is typically associated with zone 3 perisinusoidal fibrosis. Microgranulomas and lipogranulomas, sometimes quite small, are nearly always present. Portal inflammation is at least mild; it may be moderate and interface activity may be seen.

GRADE 3: SEVERE. Steatosis is usually panacinar or involves the entire regenerative nodule in cirrhotic parenchyma. Ballooning and disarray are consistently present and are predominantly in zone 3 . Lobular inflammation is increased compared with grade 2; portal inflammation is as above for grade 2 .

Table 2 shows the numerical assessments of the variables included in the grading proposal. The progressive increase in steatosis, hepatocellular ballooning, and lobular and portal inflammation is noted.

The remaining variables that were analyzed but not included in the grading system were not considered to be of significant discriminatory value for determining differences in grade; these variables were Mallory's hyaline, acidophil bodies, PAS-D Kupffer cells, glycogenated nuclei, lipogranulomas, and hepatocellular iron. Mallory's hyaline, present in only 21 of 51 biopsies, was seen in zone 3 ballooned hepatocytes; because ballooned hepatocytes are included in the system of grading, separate inclusion of hyaline in the assessment of a grade did not appear to be warranted. A trend showing more hyaline in the higher grades was seen. Although acidophil bodies and PAS-D Kupffer cells are histological indicators of cell necrosis and a trend toward increasing numbers of both was seen, reproducible quantitation is challenging and laborious, and the benefit of such quantitation is doubtful. Glycogen nuclei were present in all but seven cases; quantitation of this nuclear change likewise did not provide discriminatory information for grading of histological activity. Lipogranulomas were evaluated and

Table 2. Results of Numerical Grading of NASH Biopsies

\begin{tabular}{lcclc}
\hline \multicolumn{1}{c}{ Grade } & (n) & Steatosis* & Ballooning & Inflammation \\
\hline 1-Mild & 22 & $1-2$ & Minimal & $1-2$ acinar; 0-1 portal \\
2-Moderate & 23 & $2-3$ & Present-zone 3 & 2 acinar; 1-2 portal \\
3-Severe & 6 & 3 & Marked-zone 3 & 3 acinar; 1-2 portal \\
\hline
\end{tabular}

Table 2 shows the degrees of steatosis, ballooning, and inflammation present in the three grades of activity. The assigned numbers for steatosis are based on percent of involvement of the biopsied material by fat as stated in the methods section. Inflammation, likewise, is based on inflammatory foci per $20 \times$ field.

* Macrovesicular steatosis predominated, but, with the exception of cases with only mild steatosis in which only large droplet fat was seen, microvesicular steatosis was also present. 
Table 3. Grade and Stage of the Nine Biopsies With Stainable Iron

\begin{tabular}{clc}
\hline $\begin{array}{c}\text { Iron } \\
\text { Grade }\end{array}$ & Activity & $\begin{array}{c}\text { Stage } \\
\text { (Fibrosis Score) }\end{array}$ \\
\hline $1+$ & Mrade & 0 \\
$1+$ & Mild & 0 \\
$1+$ & Mild & 0 \\
$1+$ & Mild & 1 \\
$1+$ & Mild & 1 \\
$1+$ & Mild & 3 \\
$1+$ & Moderate & 2 \\
$2+$ & Mild & 0 \\
$2+$ & Moderate & 4 \\
\hline
\end{tabular}

Table 3 shows the activity grade and fibrosis score of each of the nine biopsies with stainable iron.

documented. Lipogranulomas were seen in varying numbers scattered throughout the lobules in 42 of 51 biopsies and in portal tracts in 3 of 51 cases, but there was no apparent relation to other indicators of histological grade. In fact, the cases with the most lipogranulomas ("several") were those with mild $(n=6)$ and moderate $(n=4)$ activity.

Nine biopsies showed iron deposition. Two biopsies had $2+$ iron, one of which was from a cirrhotic patient known to have an elevated ferritin at the time of biopsy. The second patient had normal prebiopsy serum iron studies. Correlation of grade and stage in these nine patients with iron deposition is shown in Table 3.

\section{Fibrosis Score}

The documented types and progression of fibrosis observed in the biopsies with steatohepatitis were the basis for the proposed system for staging. This system reflects both the patterns of fibrosis and the increase in connective tissue deposition and architectural remodeling noted in this series:

Stage 1. Zone 3 perisinusoidal/pericellular fibrosis; focally or extensively present.

Stage 2. Zone 3 perisinusoidal/pericellular fibrosis with focal or extensive periportal fibrosis.

Stage 3. Zone 3 perisinusoidal/pericellular fibrosis and portal fibrosis with focal or extensive bridging fibrosis.

Stage 4. Cirrhosis.

The grades of fibrosis evaluated separately (zone 3, portal, and bridging fibrosis), are shown in Table 4. Zone 3
Table 5. Correlation of Grade and Stage in NASH Biopsies

\begin{tabular}{lllcll}
\hline & \multicolumn{5}{c}{ Stage } \\
\cline { 2 - 6 } Grade & 0 & 1 & 2 & 3 & 4 \\
\hline Mild & 9 & 6 & 3 & 4 & 0 \\
Moderate & 1 & 4 & 7 & 9 & 2 \\
Severe & 0 & 1 & 0 & 4 & 1 \\
\hline
\end{tabular}

Table 5 shows the delineation of grades and fibrosis scores of the biopsies. As noted, necroinflammatory activity and stage do not necessarily correlate.

perisinusoidal or pericellular fibrosis is the initial site(s) of fibrosis in steatohepatitis and, therefore, is scored as Stage 1. This form of fibrosis persists through all the stages, and may commonly be seen in cirrhosis. Stage 2 adds the findings of various degrees of portal fibrosis; this was found to range from none in 22 cases to portal-portal bridging fibrosis in 10 cases and cirrhosis in three biopsies. Stage 3 accounts for the bridging fibrosis observed between zones 3 or zones 3 and 1 . The three stages described may show either focal or extensive involvement of the tissue represented in a biopsy; therefore, the descriptors "focal" and "extensive" are included in the staging system to formally indicate this.

Correlation of the grades and stages of the 51 biopsies is depicted in Table 5. Only moderate or severe necroinflammatory activity was seen in the three cirrhotic patients, and none of Stage 0 or Stage 2 patients showed severe necroinflammatory activity. As in chronic hepatitis, the grade and stage of disease may be disparate, as they are meant to reflect different aspects of the liver injury.

\section{Correlation With Serum Liver Enzymes}

Figure 1 shows the mean ALT and AST values for the three histological grades. The mean AST values were higher in the severe grade compared with the mild and moderate grades $(p<0.002, p<0.05)$. There were no differences between the grades in the ALT values, but a trend could be seen in both ALT and AST with higher values in the higher grades.

\section{DISCUSSION}

In current medical practice, liver biopsy is considered a necessary component in the evaluation of patients suspected of having NASH (2), and pathologists are faced with an increasing number of such biopsies to interpret. Although the histological lesions of NASH and steatohepatitis from

Table 4. Staging for Fibrosis and Architectural Remodeling in NASH

\begin{tabular}{|c|c|c|c|c|c|c|c|c|c|c|c|c|c|}
\hline \multirow{2}{*}{\multicolumn{2}{|c|}{ Stage $(n)$}} & \multirow[b]{2}{*}{ Focal/Extensive } & \multicolumn{3}{|c|}{ Sinusoidal } & \multicolumn{4}{|c|}{ Portal } & \multicolumn{4}{|c|}{ Bridging } \\
\hline & & & 1 & 2 & 3 & 1 & 2 & 3 & 4 & 1 & 2 & 3 & 4 \\
\hline 0 & 10 & 0 & & 0 & & \multicolumn{4}{|c|}{0} & \multicolumn{4}{|c|}{0} \\
\hline 1 & 11 & $10 / 1$ & 8 & 3 & 0 & 0 & 0 & 0 & 0 & 0 & 0 & 0 & 0 \\
\hline 2 & 10 & $7 / 3$ & 3 & 5 & 2 & 5 & 5 & 0 & 0 & 0 & 0 & 0 & 0 \\
\hline 3 & 17 & $4 / 13$ & 1 & 4 & 12 & 3 & 5 & 9 & 0 & 5 & 4 & 8 & 0 \\
\hline 4 & 3 & $0 / 3$ & 0 & 0 & 3 & 0 & 0 & 0 & 3 & 0 & 0 & 2 & 1 \\
\hline
\end{tabular}

Table 4 shows the distribution of the various types of fibrosis within each stage as defined in the methods section. For the scoring system proposed, Stage 1 is zone 3 perisinusoidal fibrosis, Stage 2 adds the portal component, Stage 3 includes bridging fibrosis, and Stage 4 is cirrhosis. In 10 cases, there was no fibrosis in the biopsy. 


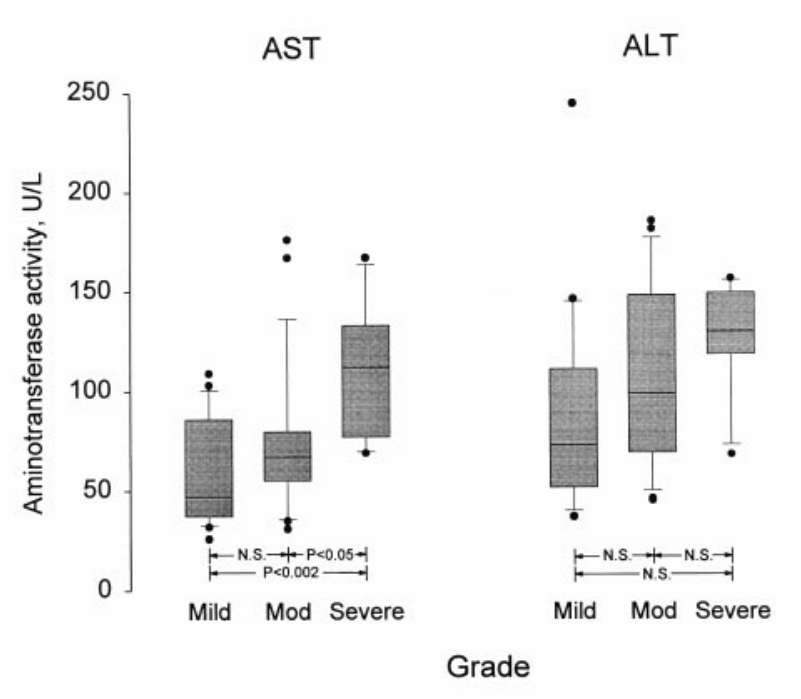

Figure 1. Box plots of the aminotransferase values and overall necroinflammatory grades. The aspartate aminotransferase (AST) levels correlated with the grade, whereas a significant correlation was not established between the alanine aminotransferase (ALT) level and necroinflammatory grade. The middle line of each box represents the median, the upper and lower ends of each box represent the 75 th and 25 th percentiles, and the single lines represent the 90th and 10th percentiles. Outliers above and below $90 \%$ and $10 \%$ are shown as data points. NS $=$ not significant by Student's $t$ test $(p>0.05)$.

other, known causes are commonly included descriptively in pathology reports, there is no system for reporting necroinflammatory activity (grade) and architectural alterations (stage) as it exists for other forms of chronic liver disease. Grading systems for chronic viral and autoimmune liver disease (10-15), the chronic cholestatic diseases PBC and PSC (16-18), and drug-induced liver disease (19) are used widely in clinical practice and in investigational studies. Although a single system for grading and staging chronic viral hepatitis is not universally used, the more recent systems advocated are modifications of the original Knodell histological activity index (10). In this system, necroinflammatory activity and fibrosis are evaluated and individual features are scored separately, but the final score is derived from adding the categories together. The newer systems assign separate scores for necroinflammatory activity (grade) and fibrosis (stage) to reflect the features of ongoing hepatocellular injury, lobular, and portal lymphocytic or lymphoplasmacytic inflammation and established portalbased fibrosis with architectural remodeling. In the chronic cholestatic diseases, PBC and PSC, histological assessment of stage is not based on hepatocyte injury and inflammatory changes but rather on bile duct lesions and the subsequent ductular proliferation and scarring that are associated with bile duct destruction and loss. In liver biopsies from psoriatic patients treated with methotrexate, a histological score for monitoring the effects of the drug in the liver is derived from a combination of hepatocellular injury and fibrosis, and is reported as a single score or stage (19).
The characteristic morphological features of steatohepatitis and NASH are steatosis, inflammation that is predominantly lobular and includes scattered polymorphonuclear leukocytes and zone 3 perisinusoidal fibrosis; these are sufficiently distinct as to preclude simply applying any one of the existing grading and staging systems. The method proposed in this study was derived to provide a system for both histopathological analysis and reporting that reflects the characteristic histological features of NASH, and is easy to apply. As with the application of any system of semiquantitative analysis, this system is intended for use only after comprehensive evaluation of the liver biopsy in the appropriate clinical setting. As others have stated (2), the evaluation should include review of more than one level by hematoxylin and eosin and special stains for connective tissue and iron. The proposed method provides a systematic approach for the evaluation and reporting of the combinations of lesions seen in steatohepatitis. The single numeric scores for grade and stage are not meant to replace the pathologist's overall assessment and evaluation of the various components that ultimately derive those scores. Figures $2 A-D$ show several of the components that comprise the constellation of features to be evaluated for grading.

The system for grading was determined after categorizing and semiquantitatively recording 10 of the known histological features of NASH $(1,7-9)$. Because all of the lesions that can be seen in NASH are not likely independently significant in reflecting the severity of ongoing injury in and of themselves, it was determined that the combination of hepatocellular steatosis, hepatocellular ballooning, and lobular inflammatory infiltrates constitute the basis for the grading scheme, as shown in Table 1.

The unique pattern of fibrosis of steatohepatitis was the basis for deciding the proposed system for staging. Zone 3 perisinusoidal/pericellular "chicken-wire" fibrosis is a common finding in liver biopsies with steatohepatitis, whether alcohol-related or NASH $(7,9)$, and most likely is a response to the steatonecrosis concentrated in this portion of the acinus. Zone 3 perisinusoidal fibrosis may be the only fibrosis present, or it may be a component in more advanced forms of fibrosis. Therefore, the proposed staging system begins with this form of fibrosis and progresses through portal fibrosis and bridging fibrosis to cirrhosis. The observed "overlap" of fibrosis in the higher stages is a reflection of the incremental deposition of collagen in the perivenular and periportal regions of the lobule with progressive injury.

Two other lesions noted in the biopsies reviewed warrant comment. Iron accumulation is known to occur in alcoholic steatohepatitis and alcoholic cirrhosis (21), although the mechanism for this is not well understood. Iron was also documented as "mild" in three of 20 biopsies in Ludwig's original study of patients with NASH (1). In our study, two of 51 biopsies showed $2+$ hepatocellular iron accumulation, seven biopsies had 1+ iron, and no hepatocellular iron was seen in 42 biopsies. Seven of the nine biopsies with elevated 


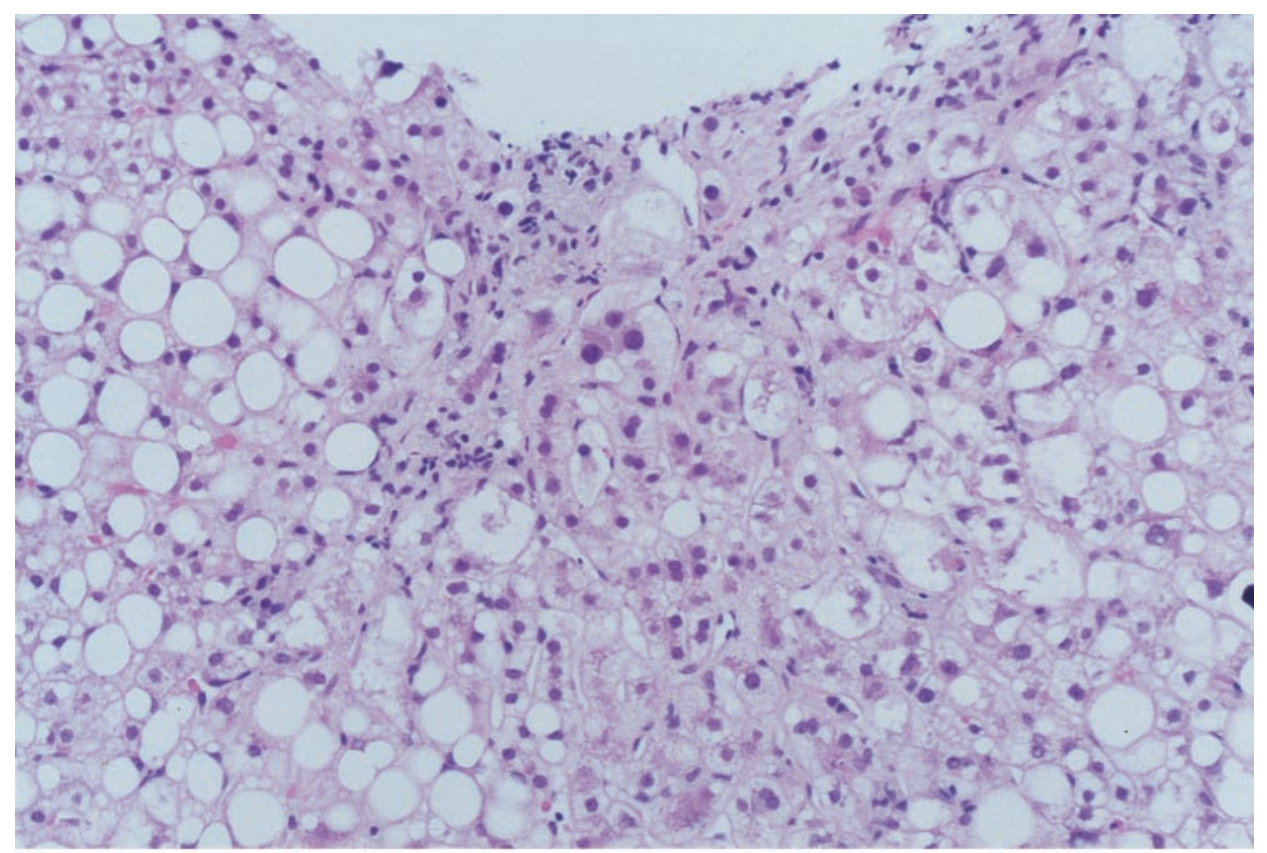

A

Figure 2A. The photomicrograph shows several of the components that comprise the constellation of features to be evaluated for grading. Steatosis is predominantly macrovesicular, ballooned hepatocytes are noted, and some contain intracytoplasmic material consistent with poorly formed Mallory's hyaline. Mild, acute, and chronic inflammation are present (hematoxylin and eosin, 20×).

stainable iron had mild activity, and four of the biopsies had no fibrosis. Table 3 shows the correlation of iron with grade and stage. Two plus iron was seen in one of the three biopsies with cirrhosis and may be an example of the iron accumulation known to occur in other, nonhemochromato- sis forms of cirrhosis (22). Patients with known hereditary hemochromatosis were excluded from this review to exclude the inflammation and fibrosis that result from sideronecrosis. The significance of the iron seen in the nine biopsies with iron is not known; it is not clear that the iron

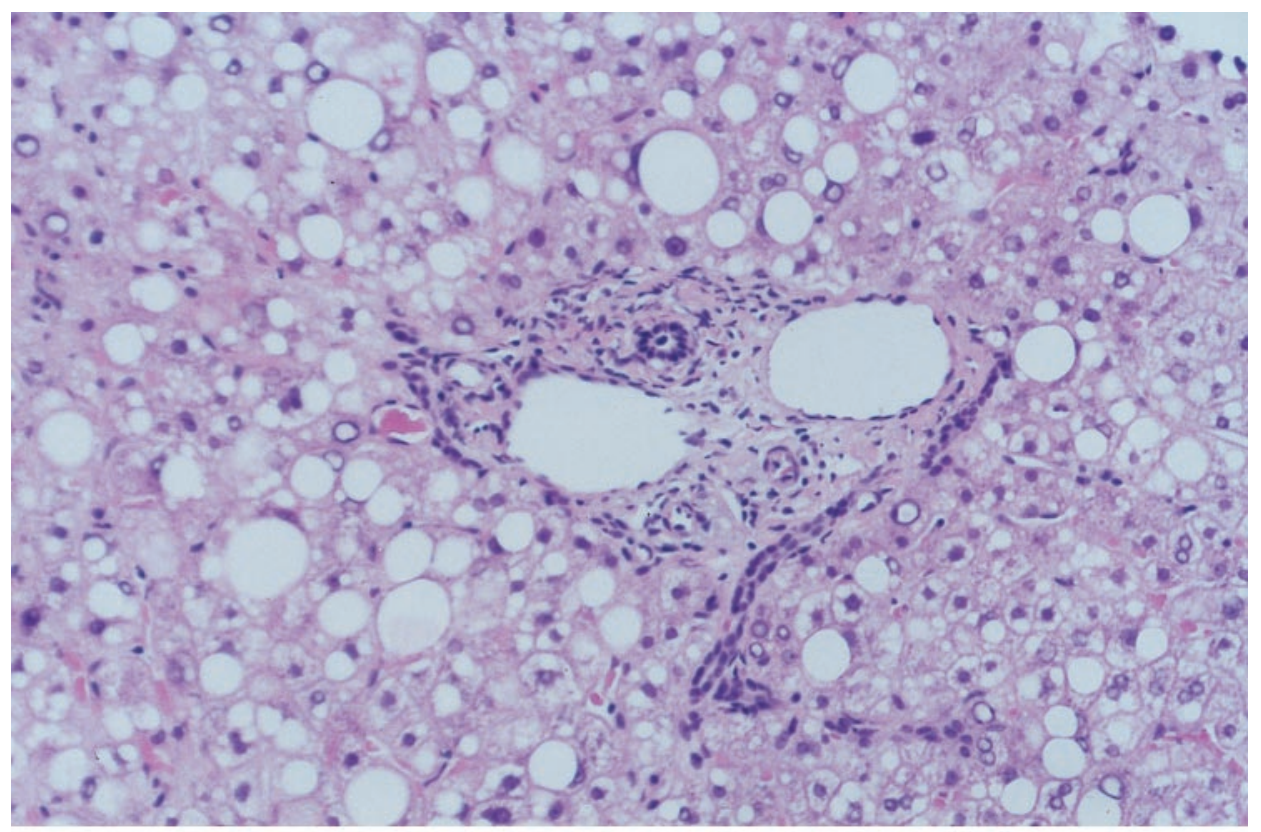

B

Figure 2B. In this field of the same biopsy, the mild portal chronic inflammation is seen. In addition, glycogenated nuclei are prominent (hematoxylin and eosin, 20×). 


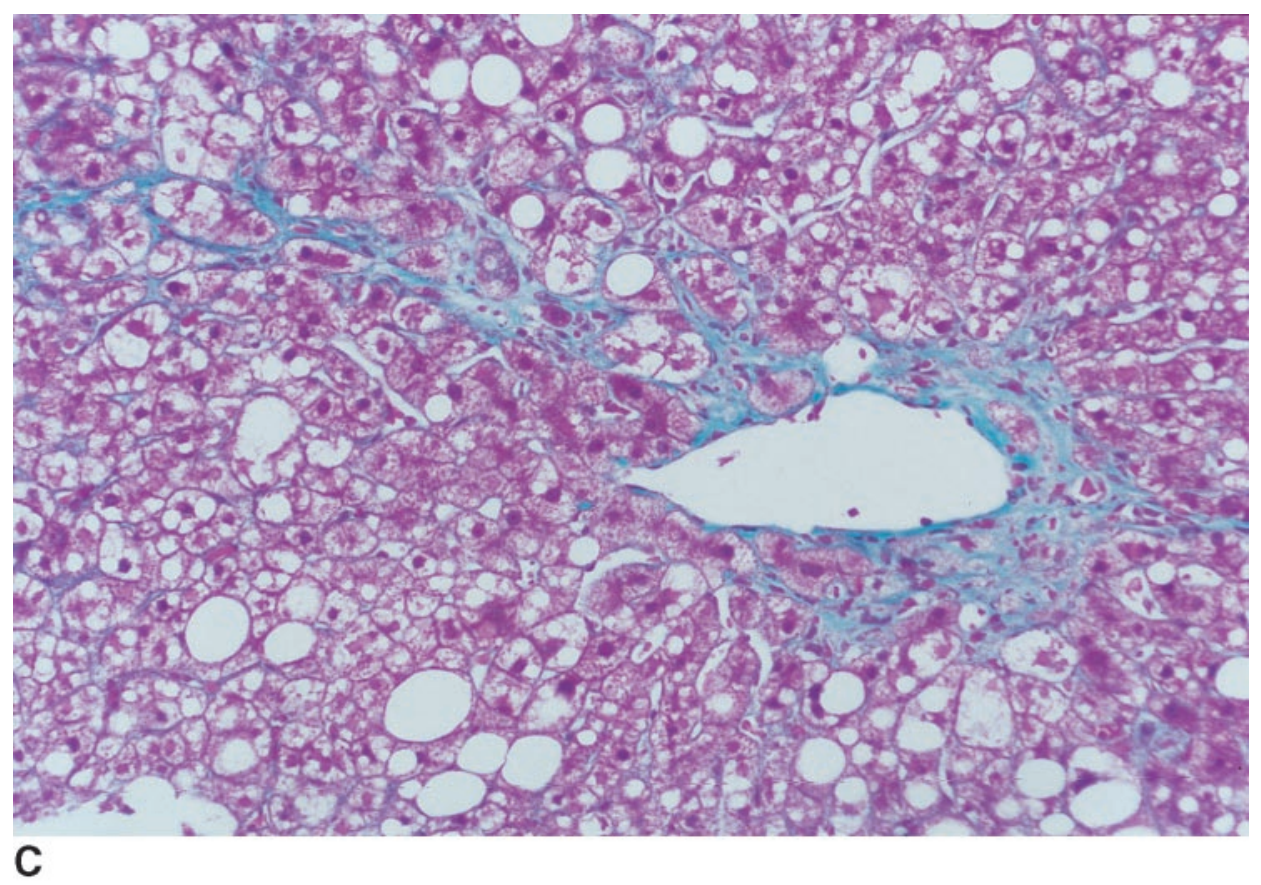

Figure 2C. The Masson's trichrome stain for collagen highlights perivenular and zone 3 perisinusoidal fibrosis, which is characteristic of the fibrosis in steatohepatitis (Masson's trichrome, 20×).

contributed to higher grades or stages in the biopsies as has been recently reported (23).

The presence of the occasional eosinophil in the portal and lobular infiltrates is not commonly reported in other studies of NASH, but, in our experience, is frequently observed with steatohepatitis and NASH biopsies. When present, a single eosinophil or occasional group of more than one may be seen in sinusoids, in microgranulomas, or in the scattered, small lipogranulomas in the lobules. Although the presence of eosinophils in liver biopsies raises the possibility of drug-related liver injury or immune response, the very few numbers of them seen and the frequency with which they are present in NASH patients with no documented medication use mitigates against this. In

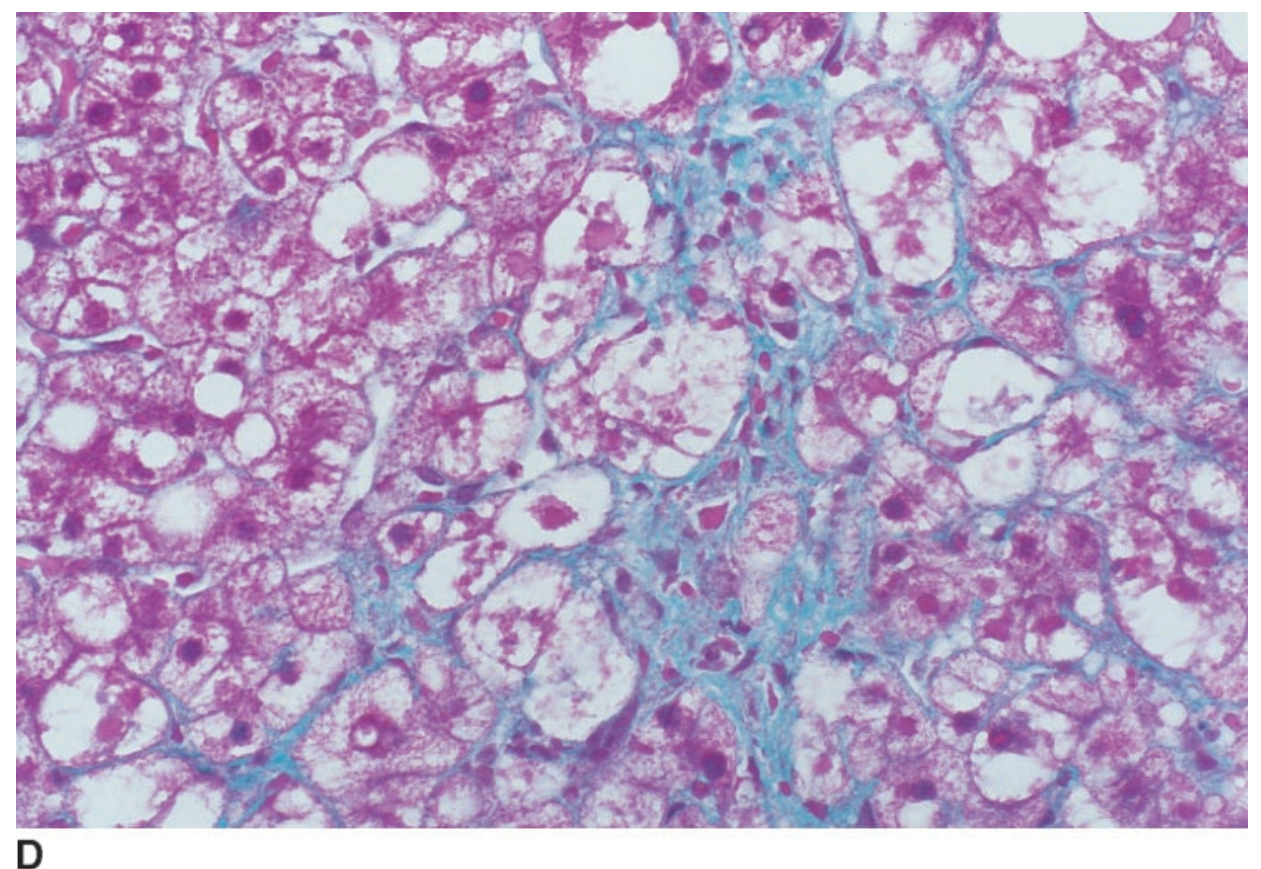

Figure 2D. In another zone 3 field, the pericellular fibrosis is noted predominantly around the ballooned hepatocytes (Masson's trichrome, $40 \times)$. 
addition, eosinophils are also known to occur in other forms of nondrug-induced liver injury including liver allograft rejection (24), chronic hepatitis $\mathrm{C}$ (25), and in primary biliary cirrhosis (16). As in the latter disease processes, the significance of eosinophils in the inflammatory component in NASH is not well understood.

The trend shown in comparing grade with ALT and AST values suggests the grading scheme may be relevant to the degree of necroinflammatory activity. However, there is notable overlap between the grades and, as with any pathological process, there is variability on an individual patient basis. The inclusion of ALT and AST values for analysis is not intended to replace careful pathological examination of a liver biopsy.

In conclusion, this retrospective study of 51 biopsies from 50 patients with NASH was undertaken to devise a system of reporting necroinflammatory grade and fibrosis stage for the purpose of standardizing the analysis and reporting of morphological features of NASH. The grading system is based on an observed progressive increase in steatosis, ballooning, and lobular inflammation. A method of staging is proposed to reflect the typical fibrosis of steatohepatitis from zone 3 perisinusoidal fibrosis to bridging fibrosis to cirrhosis. Testing the reproducibility and ease of application of this system of grading and staging, as has been done for chronic hepatitis (26), awaits future studies.

\section{ACKNOWLEDGMENT}

The authors wish to thank Dr. Robert Britton for the statistical analyses for the study.

Reprint requests and correspondence: Elizabeth $\mathrm{M}$. Brunt, M.D., Department of Pathology, Saint Louis University School of Medicine, 3635 Vista Avenue, P.O. Box 15250, St. Louis, MO 63110-0250.

Received Dec. 9, 1998; accepted Apr. 19, 1999.

\section{REFERENCES}

1. Ludwig J, Viggiano TR, McGill DB, et al. Nonalcoholic steatohepatitis. Mayo Clinic experiences with a hitherto unnamed disease. Mayo Clin Proc 1980; 55:434-8.

2. Ludwig J, McGill DB, Lindor KD. Metabolic liver disease. Review: Nonalcoholic steatohepatitis. J Gastroenterol Hepatol 1997; 12:398-403.

3. Bacon BR, Farahvash MJ, Janney CG, et al. Nonalcoholic steatohepatitis: An expanded clinical entity. Gastroenterology 1994;107:1103-9.

4. Powell EE, Cooksley WGE, Hanson R, et al. The natural history of nonalcoholic steatohepatitis: A follow-up study of forty-two patients for up to 21 years. Hepatology 1990;11:7480 .

5. Abdelmalek M, Ludwig J, Lindor KD. Two cases from the spectrum on nonalcoholic steatohepatitis. J Clin Gastroenterol 1995;20:127-30.
6. Neuschwander-Tetri BA, Bacon BR. Nonalcoholic steatohepatitis. Med Clin of N Amer 1996;80:1147-66.

7. Diehl AM, Goodman Z, Ishak KG. Alcohol-like liver disease in nonalcoholics. A clinical and histologic comparison with alcohol-induced liver injury. Gastroenterology 1988; 95: 1056-62.

8. Lee RG. Nonalcoholic steatohepatitis: A study of 49 patients. Hum Pathol 1989; 20:594-8.

9. Itoh S, Yougel T, Kawagoe K. Comparison between nonalcoholic steatohepatitis and alcoholic hepatitis. Am J Gastroenterol 1987;82:650-4.

10. Knodell RG, Ishak KG, Black WC, et al. Formulation and application of numerical scoring system for assessing histological activity in asymptomatic chronic active hepatitis. Hepatology 1981;1:431-5.

11. Ludwig J. The nomenclature of chronic active hepatitis: An obituary. Gastroenterology 1993:105:274-8.

12. Desmet VJ, Gerber M, Hoofnagle JH, et al. Classification of chronic hepatitis: Diagnosis, grading and staging. Hepatology 1994;19:1513-20.

13. Ishak KG. Chronic hepatitis: Morphology and nomenclature. Modern Pathol 1994; 7:690-713.

14. Scheuer PJ. Classification of chronic viral hepatitis: A need for reassessment. J Hepatol 1991;13:372-4.

15. Ishak KG, Baptista A, Bianchi L, et al. Histological grading and staging of chronic hepatitis. J Hepatol 1995;22:696-9.

16. Ludwig J, Dickson ER, McDonald GS. Staging of chronic non-suppurative destructive cholangitis (syndrome of primary biliary cirrhosis). Virchows Arch Pathol Anat 1978; 379:10312.

17. Scheuer P. Primary biliary cirrhosis. Proc R Soc Med 1967; 60:1257-60.

18. Ludwig J, LaRusso NF, Weisner RH. Primary sclerosing cholangitis. In: Peters RC, Craig JR, eds. Contemporary issues in surgical pathology, Vol. 8. Liver pathology. New York: Churchill Livingstone, 1986:193-213.

19. Roenigk HH, Auerbach R, Mailbach HI, et al. Methotrexate guidelines-revised. J Am Acad Dermatol 1982;6:145-55.

20. Searle J, Kerr JRF, Halliday, et al. Iron storage disease. In: MacSween RNM, Anthony PP, Scheuer PJ, Burt AD, Portmann BC, eds. Pathology of the liver. London: Churchill Livingstone, 1994:224.

21. Irving MG, Halliday JW, Powell LW. Association between alcoholism and increased hepatic iron stores. Alcohol Clin Exp Res 1988;12:7-3.

22. Ludwig J, Hashimoto E, Porayko MK, et al. Hemosiderosis in cirrhosis: A study of 447 native livers. Gastroenterology 1997; 112:882-8.

23. George DK, Goldwurm S, Macdonald GA, et al. Increased hepatic iron concentration in nonalcoholic steatohepatitis is associated with increased fibrosis. Gastroenterology 1998; 114:311-8.

24. Snover DC, Sibley RK, Freese DK, et al. Orthotopic liver transplantation: A pathological study of 63 serial liver biopsies from 17 patients with specific reference to the diagnostic features and natural history of rejection. Hepatology 1984;4: 1212-22.

25. Mattia AR, Ross MA, Trainer TD. Eosinophils and hepatitis C. Mod Pathol 1997; 10:146A (abstract).

26. Goldin RD, Goldin JG, Burt AD, et al. Intra-observer and inter-observer variation in the histopathological assessment of chronic viral hepatitis. J Hepatol 1996; 5:649-54. 